\title{
TRANS-
}

Revue de littérature générale et comparée

\section{Zones de défection temporaire. Refigurations de Bartleby et de des Esseintes dans quelques romans contemporains}

Justine Huppe and Frédéric Claisse

revues.org

Electronic version

URL: http://trans.revues.org/1269

ISSN: 1778-3887

\section{Publisher}

Presses Sorbonne Nouvelle

Brought to you by Université de Liège

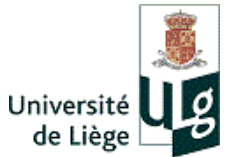

Electronic reference

Justine Huppe and Frédéric Claisse, «Zones de défection temporaire. Refigurations de Bartleby et de des Esseintes dans quelques romans contemporains », TRANS- [Online], 20 | 2016, Online since 14 October 2016, connection on 10 November 2016. URL : http://trans.revues.org/1269; D0I : 10.4000/ trans. 1269

This text was automatically generated on 10 novembre 2016.

Tous droits réservés 


\title{
Zones de défection temporaire. Refigurations de Bartleby et de des Esseintes dans quelques romans contemporains
}

\author{
Justine Huppe and Frédéric Claisse
}

\section{Last Exit to Nulle Part}

Problème : Si le récit est devenu l'un des principaux outils de domination et d'instrumentalisation des corps, des consciences et des compétences, que faire, qu'en faire? S'en éloigner? S'en foutre? S'en saisir? Le retourner contre lui-même ${ }^{1}$ ?

Cette inquiétude qui saisit Jean-Charles Massera face au pouvoir et au devenir du récit est aujourd'hui celle de nombreux écrivains, qui ressentent que leur instrument de travail, dénaturé en storytelling, leur a été confisqué par des professionnels de la communication, de la politique ou de l'entreprise, à des fins de contrôle et de manipulation ${ }^{2}$. À quoi bon continuer d'investir la forme narrative, quand l'espace public est déjà saturé de fictions et de récits ? Quelle résistance la littérature peut-elle encore offrir, et avec quels moyens, quand ses pouvoirs semblent lui avoir été confisqués?

2 Le plaidoyer de Massera pour la recherche de nouvelles formes de représentation littéraire en prise sur leur époque, s'ouvre sur un "préambule à une critique perdue d'avance ", dont l'énoncé renvoie implicitement au thème du «retard» ou du " désarmement de la critique ", au cœur de l'important ouvrage de Luc Boltanski et Ève Chiapello sur l'évolution du capitalisme ${ }^{3}$. Le retard de la littérature dans sa fonction critique - retard des formes disponibles pour dire le monde - serait une modalité de 
l'assèchement plus général des ressources critiques pour exprimer son indignation devant le désenchantement et l'aliénation, la misère et l'égoïsme, qui accompagnent depuis deux siècles le processus d'accumulation du capital. Prises de vitesse, la critique et la littérature restent attachées à des formes de lutte et d'expression que la logique capitaliste a déjà récupérées à son profit. Loin de représenter une force d'opposition, les exigences de créativité, d'authenticité et d'innovation, qui constituaient le ressort de la critique " $\operatorname{artiste}^{4}$ », ont régénéré en profondeur le capitalisme au point de former l'ossature de son «nouvel esprit ».

Dans la phase récente de ce processus, le storytelling, cet " art de raconter des histoires ", se rapproche de celui de " conduire les conduites » - un " pouvoir de scénarisation " par lequel suivre une histoire, pour un individu, signifie se faire embarquer dans une séquence d'actions déjà prévue, scriptée par autrui ${ }^{5}$. "À la place des chaînes de montage, des engrenages narratifs ${ }^{6} . »$ Dans l'imaginaire contemporain, le monde est devenu une histoire, un jeu, indiscernable des univers produits par la fiction elle-même ${ }^{7}$. Or, si plus rien n'échappe à la fictionnalisation généralisée, si la fiction ne constitue plus une force d'opposition, mais une injonction participant de la logique même du système qu'elle dénonce, quelles options s'offrent encore aux écrivains?

Pour cerner les stratégies mises en place par les auteurs dans une société où fictions et récits circulent en continu, on peut recourir à la typologie élaborée par le politologue Albert Hirschman ${ }^{8}$. Insatisfaits face à ce qu'ils perçoivent comme une baisse dans la qualité d'un produit ou d'un service, les agents sociaux ou économiques auraient trois possibilités : exprimer publiquement leur mécontentement («voice», prise de parole); abandonner le produit ou le service en question au profit d'un autre (« exit », défection); ou choisir de s'y attacher malgré tout («loyalty»). Soit, en rapport avec notre objet: certains auteurs protestent contre la mainmise du monde marchand sur le récit et l'imaginaire en s'en faisant le témoin ou en la dénonçant (voice $)^{9}$. Mais d'autres préfèrent la défection (exit): arrêter de produire de la fiction littéraire, changer de support d'expression, voire cesser carrément de croire en un quelconque pouvoir de la littérature ${ }^{10}$. Non moins lucides, un dernier groupe, sans doute majoritaire, prend acte des mutations qui affectent le champ littéraire, mais conserve un rapport de fidélité inquiète à la littérature narrative de fiction, privilégiant un «faire avec " plutôt qu'un «faire contre $^{11} »$ (loyalty).

5 L'originalité de la situation actuelle tient à ce que tant les possibilités de voice que d'exit semblent exténuées. La critique et la défection supposent en effet le recours à un autre principe au nom duquel dénoncer la situation présente. Or, c'est bien le branchement sur une extériorité de ce type qui fait aujourd'hui le plus défaut à ceux qui s'indignent de la récupération du récit à des fins instrumentales. Les appels à fabriquer des contrescénarisations ou des contre-fictions ${ }^{12}$ rebondissent comme sur une paroi molle. Pour le dire autrement, il n'y a plus de « dehors ».

6 Prenons un exemple. Croyant trouver une terre d'exil dans le monde vidéoludique des Sims, Chloé Delaume comprend qu'elle n'a finalement fait qu'entrevoir le reflet de son propre univers, les Sims étant « ce qui arrive quand tout est capital ${ }^{13}$ ». S'incarnant dans l' universims, elle y mène une enquête ethnographique, fière des avantages de sa nouvelle condition (revenus illimités, simplicité du langage, maîtrise du temps, émotivité modérée, etc.). Mais peu à peu lui apparaissent les désavantages de cette vie en réseau :

Dans le monde réel, on peut avoir la paix lorsqu'on reste chez soi. Ici, impossible de se vouer à ses plants de tomates en toute quiétude, de lire son journal en terrasse, 
de baguenauder autour de sa piscine privée ou de se concocter de petits hamburgers sans que surgissent soudain des voisins fort avides de communication. C'est assez perturbant. Sans compter la pression. Le logiciel veut notre bien. Il connaît les bienfaits d'un réseau foisonnant. Le logiciel veut notre bien ${ }^{14}$.

7 Au fur et à mesure de l'ouvrage, les passages métaleptiques entre la réalité et l'universims se multiplient. Chloé Delaume se retrouve alors prisonnière d'une sorte de panfictionnalisme ${ }^{15}$, puisqu'elle finit par admettre que l'univers réel est aussi innervé de fictions que la fiction elle-même. Son prétendu exil ne lui permet donc pas de fuir le monde contemporain, mais seulement de se confronter à ses mécaniques les mieux rodées.

8 À peine exprimée, la critique est déjà endogénéisée - comme l'exprime Octave Parrango, le personnage de 99 Francs, depuis son poste d'observation de créatif publicitaire désabusé :

Je souris parce que, si ça se trouve, dès que ce livre sortira, au lieu d'être foutu à la porte, je serai augmenté. Dans le monde que je vais vous décrire, la critique est digérée, l'insolence encouragée, la délation rémunérée, la diatribe organisée. Bientôt on décernera le Nobel de la Provoc et je ferai un candidat difficile à battre. La révolte fait partie du jeu ${ }^{16}$.

Pourtant, à sa manière, Octave résiste. Du niveau des écrivains, la quête d'une extériorité critique s'est transportée dans le roman contemporain, devenu un terrain de lutte sur lequel des personnages, face à l'empire de la fiction, explorent des possibilités d'expression de leur désaccord ou des tentatives de défection.

Ce sont ces dernières que nous étudierons dans cet article, à travers quelques figures contemporaines (chez des auteurs comme Emmanuelle Pireyre, Aurélien Bellanger, Mika Biermann ou Philippe Vasset), qui adoptent une même stratégie de repli, à l'écart du monde, pour éprouver un autre rapport à la production et à la consommation de fictions ${ }^{17}$ . Ainsi retranchés, nos personnages semblent renouer avec la posture de Bartleby, pour son refus tranquille d'obéir aux injonctions sociales ( I I would prefer not to ») ou de des Esseintes, dans son désir d'esthétiser son existence («Anywhere out of the world»). Il y a dans la fascination contemporaine pour le scribe de Melville ${ }^{18}$, comme il y eut dans la recherche d'une homologie entre les deux «fins-de-siècle", le signe d'une quête désespérée d'une figure enfin capable de s'arracher, radicalement et sans compromis, d'un monde qui semble ne plus offrir d'autre issue que déjà spectacularisée, contaminée par la fiction. Parce qu'ils continuent de participer de l'imaginaire de ce début de siècle, ces (anti-)héros littéraires $\mathrm{du} \mathrm{xIX}^{\mathrm{e}}$ siècle représentent, avec quelques autres que nous introduirons au fil de l'analyse, des figures-limites à l'aune desquelles nous interrogerons les personnages de notre corpus contemporain.

\section{Un espace structural de possibilités d'exit}

Cette comparaison passe par la construction d'un espace de variations qui permet à la fois d'opposer les personnages et de mettre en évidence les traits communs de leurs mondes un espace structural de positions et de possibilités. De quoi s'échappe-t-on, hier et aujourd'hui? Quelles ressources sont nécessaires pour organiser sa fuite avec succès? Que laisse-t-on derrière soi et à quelles conséquences s'expose-t-on?

11 Nous ferons l'hypothèse qu'entre le XIX ${ }^{e}$ siècle et le début du XXI ${ }^{e}$, quelque chose a changé qui ne garantit plus la possibilité de faire défection. La possibilité même d'un "dehors » 
est devenue problématique. L'attitude de Bartleby ou de des Esseintes exprimait encore, en creux ou en relief, une distance critique à l'égard du monde. Nettement moins polémiques, les figures, plus récentes, des geeks, nerds et autres nolifes ${ }^{19}$ de notre corpus représentent des cas de résistance minimale aux insatisfactions du réel, par l'aménagement de "bulles » dont l'extraterritorialité, d'ailleurs toute relative, s'avère tout à fait fonctionnelle, adaptée aux sollicitations du monde. On peine à y trouver des figures qui accèdent à une forme de vie différente, véritablement autonome. Nos personnages rêvent d'arrachement, de conquête d'un dehors, mais il s'agit le plus souvent de « zones de défection temporaire », pour mieux revenir ensuite dans le monde.

Un détour par l'essai de Hakim Bey, dont nous détournons ici le titre ${ }^{20}$, permettra de mieux percevoir l'enjeu de ces défections. Inspirées par le modèle des utopies pirates, les «zones d'autonomie temporaire " sont encore très marquées, lors de leur parution en 1991, par la figure émergente du réseau, dans laquelle l'auteur perçoit un potentiel de subversion qui ne cessera ensuite de nourrir l'imaginaire de la cyberculture et des mouvements altermondialistes. Décentralisés, souterrains, invisibles, capables de se reconfigurer au gré des besoins - d'activer ces zones comme on mène des « opérations de guérilla » avant de les dissoudre pour ensuite les reformer ailleurs - ces espaces ont pour adversaire l'État - «l'État terminal, l'État de l'information méga-entrepreneurial, l'empire du Spectacle et de la Simulation ", avec qui il est hors de question d'envisager un «choc frontal» révolutionnaire. Temporaires à défaut de renverser radicalement la situation et d'aboutir à un changement permanent, les zones représentent néanmoins des " expériences maximales », des moments de très grande intensité qui « donnent forme et sens à la totalité d'une vie ». Or, si l'empire du Spectacle et de la Simulation n'a guère changé, il a depuis lors adopté la forme même du réseau que Hakim Bey pensait encore pouvoir lui opposer il y a vingt-cinq ans. Quant à la temporalité insurrectionnelle des zones temporaires, elle s'est aisément traduite dans celle du "projet», qui forme aujourd'hui la norme de carrière intériorisée par les individus et les entreprises. Ce qui représentait encore une extériorité accessible en 1991 s'est progressivement transformé en modèle dominant non seulement de notre participation au monde, mais de nos tentatives pour nous en échapper.

\subsection{Axe 1 : critique vs. compromis}

13 S'opposer au monde en réseau et à la "cité par projets ", mais en en passant par la «formule d'investissement » au fondement des « relations naturelles entre les êtres » qui l'habitent (l'autonomie, la flexibilité, la déterritorialisation du « nomade»), relève de ce que le sociologue Luc Boltanski, dont nous reprenons ici à nouveau les termes, appellerait une figure de compromis ${ }^{21}$. La plupart des figures de notre corpus contemporain relèvent de ce cas de figure. Au compromis, on peut opposer la critique, à laquelle nous avons déjà associé les personnages de Bartleby et de des Esseintes. Ensemble, critique et compromis forment les deux pôles d'un premier axe sur lequel se distribuent nos personnages en quête d'exit.

14 À l'évidence, sauf anachronisme, l'activité critique de personnages du XIX ${ }^{\mathrm{e}}$ siècle ne peut porter sur le « monde en réseau », mais sur le système de "grandeurs » (ou de valeurs) de leur temps, qu'ils dénoncent, sans concession, au nom d'un autre ensemble de principes. Par son isolement volontaire, le duc Jean des Esseintes se soustrait tant au système de production économique (que lui permet son statut de rentier) que de reproduction (il 
opte pour le célibat). Mais ce héros négatif n'en est pas moins un créateur : c'est par ses rejets et mépris que sa subjectivité s'hypertrophie et s'affirme contre la médiocrité du commun. Il opère donc à la fois une critique radicale de l'échelle des valeurs dominantes (productivité, efficacité, progrès social, transmission d'un nom ou d'un patrimoine) pour leur en opposer d'autres - créativité, inspiration, authenticité, dont la valeur de contestation est alors encore pleine et entière. Une même radicalité nourrit l'expérience de Bartleby. C'est au cœur du monde, en plein Wall Street, qu'il se confine dans des espaces restreints (le bureau, la prison) et dans les mots, refusant poliment, mais avec résolution, de travailler. C'est là toute sa force: muré dans un refus presque mutique, Bartleby ne donne aucune prise. À la différence de des Esseintes qui dispose d'un point d'appui normatif extérieur (l'idéal d'une vie esthétisée), Bartleby fait surgir le conflit en s'abstenant d'affirmer des valeurs. Mais d'une certaine manière, son extériorité est ainsi maximale, puisqu'il se soustrait à l'impératif de justification.

À l'opposé de ces figures critiques, leurs (quasi-)contemporains Oblomov et Emma Bovary représentent des figures de compromis avec leur temps. À l'instar de des Esseintes, Oblomov est un jeune rentier. Mais contrairement au héros de Huysmans, celui de Gontcharov est moins un esthète de l'existence qu'un homme épris de quiétude. Si sa paresse chronique et sa phobie de l'affairisme l'empêchent de satisfaire aux attentes de la société de son temps en termes de production (incapacité à administrer ses affaires) et de reproduction (abandon d'un projet de mariage, choix d'un moins bon parti, progéniture sans consécration matrimoniale), il semble que ce soit malgré lui: Oblomov n'a pas renoncé au rêve d'être un propriétaire, un père et un mari heureux. Il manifeste à plusieurs reprises son besoin de distinction, comme dans cette scène où il dispute son serviteur qui avait osé suggérer qu'ils étaient des gens comme «les autres ${ }^{22}$ ». Emma Bovary peut aussi, de ce point de vue, être considérée comme une figure « ajustée » à son monde. Captive du mariage, de la maternité et de la province, elle se réfugie dans les mondes que déploient la fiction et les rêves. Si elle ne vit pas recluse, elle affectionne cependant les mondes clos (le couvent, le confessionnal, la chambre), mais, comme Oblomov, reste désireuse de maintenir les apparences, en faisant de cette deuxième vie, où elle se consume autant en passion amoureuse qu'en achats compulsifs, une vie cachée, dont elle anticipe le dévoilement en se donnant la mort.

\subsection{Axe 2 : état de grand vs. état de petit}

16 Si chacun de nos personnages éprouve des difficultés à « faire société » et tente à sa façon de la déserter, la manière dont ils se justifient nous renseigne sur les normes qu'ils transgressent et sur celles qu'ils défendent. Dans De la justification, Boltanski et Thévenot ont identifié la pluralité d'échelles de grandeurs disponibles sur lesquelles s'appuie la critique $^{23}$. Ces grandeurs sont orientées en fonction d'un principe de bien commun, que les auteurs appellent "cités». Nos personnages du XIx siècle s'enracinent dans un contexte où dominent les cités « industrielle » et « domestique ». Dans la cité industrielle prévalent les principes d'efficacité, de productivité et d'entrepreneuriat. La cité domestique s'organise quant à elle autour de valeurs traditionnelles - la famille, la hiérarchie, le respect des normes et des conventions. Plus exactement, ces romans permettent d'illustrer ce que Boltanski et Chiapello appelleront le "premier esprit du capitalisme", fait d'une combinaison de valeurs bourgeoises à la fois novatrices (efficacité et travail) et traditionnelles (héritage et lignée) ${ }^{24}$. 
17 Or, nos figures d'inactifs et d'improductifs questionnent cet ordre de grandeur. Tous subissent en conséquence une forme de sanction sociale pour leurs tentatives de défection : réprimandes de Stolz envers son ami Oblomov, désarroi mêlé de charité du patron de Bartleby, impasse financière dont personne n'aide Emma Bovary à se sortir. Quant à des Esseintes, il a non seulement tout mis en œuvre pour se mettre à l'abri d'une sanction, mais imagine de surcroît un cruel dispositif qui le vengera de la société qu'il a fui. Puisque la norme sociale vise la meilleure allocation de moyens en vue d'une fin, il a l'idée de fabriquer, au nom de ce principe, un criminel - le jeune Auguste Langlois, à qui il fait subir la douloureuse expérience de vivre au-dessus de ses moyens, dans l'espoir qu'après avoir pris goût à une vie de luxe, il doive voler et tuer pour en jouir à nouveau ${ }^{25}$. Notons qu'outre cette sanction - interne aux textes - la réception a eu tendance à faire de ces personnages des figures pathologiques, réaffirmant ainsi la norme en en marquant les déviances. Néanmoins, chez nos deux rentiers, cette déviance est compensée par la possession de ressources qui leur permettent d'atteindre temporairement ou définitivement leur objectif. Si des Esseintes peut se consacrer à ses activités d'esthète, c'est parce qu'il a les moyens, symboliques et réels, de se passer de la communautée ${ }^{26}$. Oblomov ne peut se permettre d'adopter une posture aussi radicale ; néanmoins, les choix qu'il pose sont des compromis qui visent à s'assurer des rentrées d'argent suffisantes pour maintenir son idéal d'ataraxie. Le point essentiel réside ici: tous deux restent cependant "grands » à leur manière : Oblomov parce qu'il refuse de sacrifier son statut d'aristocrate russe, des Esseintes parce que, même déchu de ce qui fait la grandeur dominante de son époque, il parvient à reconstituer un univers privé sur un mode critique, au nom d'une autre grandeur, celle de la cité « inspirée », où il peut exprimer sa créativité et sa singularité.

Le manque de ressources financières nuit par contre radicalement aux fuites hallucinées d'Emma Bovary. Endettée et honteuse, elle est avant tout entravée par sa dépendance économique à l'égard de Charles. De même, Bartleby ne jouit d'aucun confort matériel. Retranché derrière son impénétrable réplique, il ne nous permet cependant pas de savoir si son refus de la justification traduit l'échec d'une tentative d'exit, faute de disposer des moyens adéquats, ou le succès paradoxal d'un projet de refus radical qui trouverait son aboutissement dans la mort et l'emprisonnement. À nouveau, Bartleby pervertit nos schémas puisque l'«état de petit» (manque de moyens, refus du travail) qu'il partage avec Emma peut être renversé en grandeur (résolution, fermeté de l'entreprise critique), lecture à laquelle souscrit le plus souvent la critique des $\mathrm{xx}^{\mathrm{e}}$ et $\mathrm{xxI}^{\mathrm{e}}$ siècles qui a redécouvert le personnage.

Au XIX ${ }^{e}$ siècle, pour aboutir, le désir de se mettre hors jeu doit donc aller de pair avec des capacités économiques. Paradoxalement, s'extraire des systèmes de production et/ou de reproduction nécessite de bénéficier d'une accumulation de ce qui ordonne les grandeurs de ce même système : en l'occurrence, l'argent. 


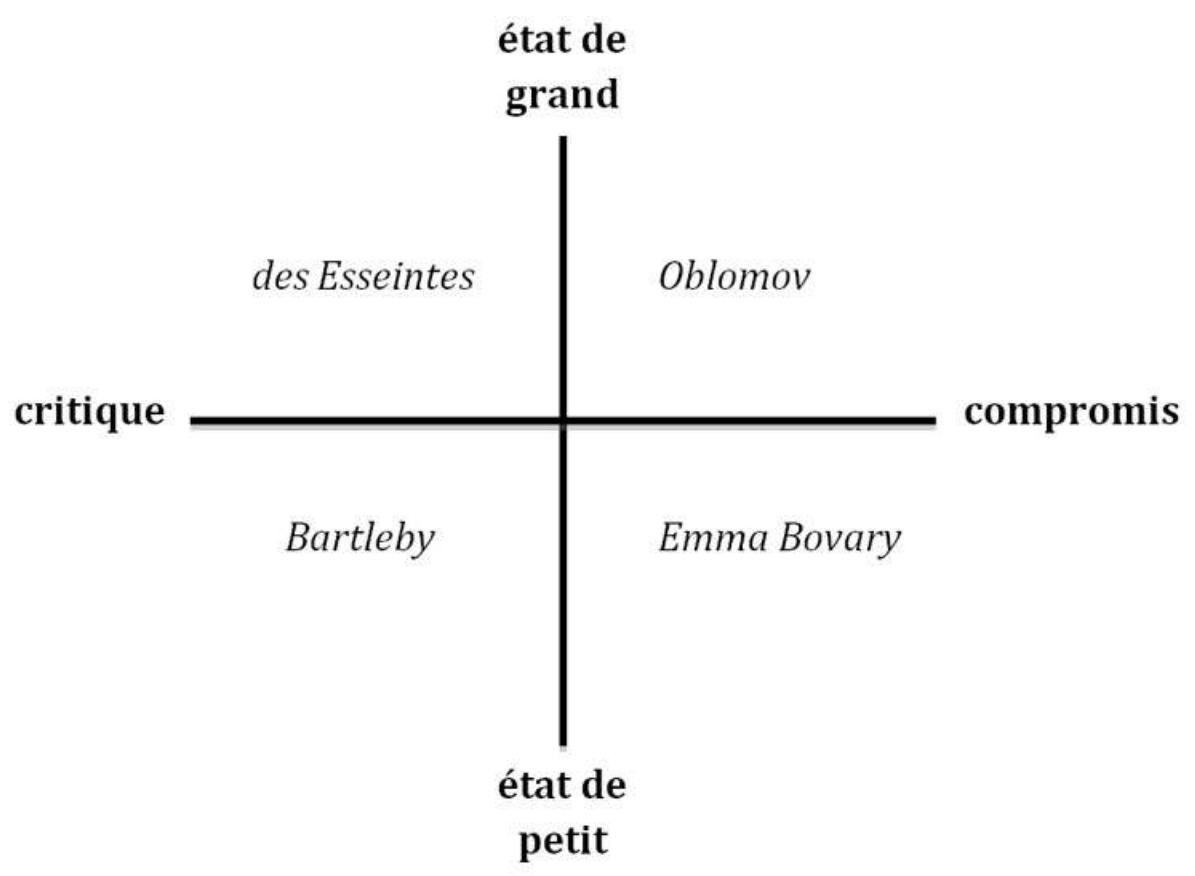

\section{La figure de l'homme qui dort (Perec) et le deuxième esprit du capitalisme}

Aux xxe et $\mathrm{xxI}^{\mathrm{e}}$ siècles, les grandeurs ont changé. À cet égard, Un homme qui dort (Georges Perec), qui peut être lu comme une sorte de réécriture de la nouvelle de Melville ${ }^{27}$, représente une charnière importante, entre le deuxième esprit du capitalisme (fondé sur la rationalité bureaucratique de la grande entreprise et sa contribution à la justice sociale) et son nouvel esprit, basé sur la capacité à s'adapter, à inventer et à étendre un réseau de liens.

21 Le personnage de Perec est pourtant très différent de celui du scribe. Ne se retirant pas du système de production, puisqu'il continue de consommer, il adopte moins une posture de refus que d'indifférence, de recherche d'une vie "neutre». Cultivant longtemps l'indécision quant à ce que sera sa vie future, le jeune homme choisit de freiner au moment où sa vie devrait s'engager sur des rails. Enfin, au contraire de Bartleby, qui pousse son projet jusqu'à ses conséquences les plus extrêmes, l'homme qui dort ne mène qu'une expérience temporaire, qui lui permet de mieux revenir au monde.

Certains aspects du roman, comme la lassitude identitaire du personnage et le peu d'importance accordé à son statut économique, annoncent ainsi certaines caractéristiques de notre corpus contemporain. D'une certaine manière, la critique adressée par le héros de Perec au monde social qu'il refuse d'intégrer sera entendue : à ce sentiment d'aliénation et d'inauthenticité, à l'ennui d'une vie toute tracée, le capitalisme saura proposer des motifs d'engagement qui puisent au contraire dans la subjectivité et la créativité de l'individu. 


\section{4. $\mathrm{XXI}^{\mathrm{e}}$ siècle et monde en réseau : No Way Out?}

Dans un monde en réseau, ce qui fait la grandeur des individus est leur capacité à s'engager dans des activités, à faire preuve de flexibilité et de polyvalence pour passer avec succès d'un projet à l'autre. Or, s'il répond aux critiques d'inauthenticité et d'hyperrationalité adressées au deuxième esprit du capitalisme (dont témoigne Un homme qui dort), le nouvel esprit associé au monde en réseau se révèle en définitive encore plus éreintant pour l'individu, éprouvé par la succession de projets, qui l'obligent à un incessant développement de lui-même pour maintenir son employabilitée ${ }^{28}$. Insécurisé et incapable de répondre à ces injonctions, le sujet contemporain se réfugierait dans des formes d'effacement provisoire. La dépression, cette «fatigue d'être soi » de l'individu en panne, correspond bien, comme le dit Alain Ehrenberg, à la " pathologie d'une société où la norme n'est plus fondée sur la culpabilité et la discipline mais sur la responsabilité et l'initiative ${ }^{29}$ ». David Le Breton s'interroge quant à lui sur les individus en quête de "blancheur " (sommeil, burn out, immersion dans une activité dérivative, fuite dans le virtuel, errances, anorexie, etc. $)^{30}$.

En étudiant un corpus de deux cents romans publiés entre 1968 et 2004, Anne Barrère et Danilo Martucelli ${ }^{31}$ ont formulé des observations qui corroborent ces analyses sociologiques des effets du « nouvel esprit du capitalisme » sur la subjectivité. Se mettrait en place un nouveau dispositif de saisie des personnages littéraires basé sur l'énergie. La littérature interrogerait moins les frontières entre l'individu et le monde que la manière dont s'investissent et se connectent des personnages allégés des propriétés sociales ou des traits psychologiques qui sclérosaient leur identité. Les intrigues se focaliseraient davantage sur leurs variations d'énergie (pannes, courts-circuits, explosions, etc.). Barrère et Martuccelli ont ainsi distingué quatre régimes d'énergie, parmi lesquels celui des « arrachements et des fuites » devant la suractivité du monde contemporain.

Quelles formes prend cette fuite pour les personnages contemporains de notre corpus geeks, artistes en recherche d'isolement, militants autonomes et autres ermites des mondes virtuels qui cherchent à se soustraire à la surabondance des liens? Quels moyens suppose-t-elle ? Plus profondément : la fuite est-elle seulement toujours possible dans un monde en réseau?

\subsection{Axe 2 : état de grand / état de petit : les « mailleurs » contre les "faiseurs »}

Demandons-nous d'abord quelles grandeurs sont en jeu dans ces tentatives d'exit. Selon le principe de justification qui ordonne la «cité par projets» dans un monde en réseau, le " grand » est un " mailleur ", c'est-à-dire un individu capable de faire croître le réseau en tissant des liens, à l'opposé de celui qui en capte et consomme les ressources à son seul profit (qualifié de «faiseur »)32. Compte tenu des récents ressourcements du capitalisme par la fiction, le virtuel et les cultures participatives, il n'est pas étonnant que la créativité fasse partie des compétences reconnues chez un mailleur. Nous en trouvons de nombreuses illustrations dans notre corpus.

La fiction en réseau fournit une échappée satisfaisante à certains personnages, surtout quand elle permet de démontrer leurs qualités de mailleur. C'est ainsi que la jeune 
Batoule, extraite de la galerie de personnages hyperconnectés de Féerie générale (Emmanuelle Pireyre), parvient à s'évader de sa condition ordinaire de collégienne musulmane à travers la modération d'un forum Internet consacré à la rédaction de fanfictions. Comme d'autres rédactrices assidues, elle considère que la fiction est un espace de reconquête de soi. Emmanuelle Pireyre s'inquiète cependant de la ligne de défense de ces jeunes auteures, qui rejoint celle des gourous du storytelling ${ }^{33}$, signe que la fiction n'est plus un véritable dehors.

Dans La Carte et le territoire, Michel Houellebecq entrecroise les destins de Jed Martin, un artiste contemporain, et de son propre personnage d'écrivain. Partageant une propension à l'isolement et, paradoxalement, une position stratégique sur le marché de l'art qui font d'eux des connecteurs incontournables, les deux artistes accumulent cependant les ressources qui leur permettent de vivre retranchés, loin de Paris, ses galeries et ses sociabilités: Houellebecq en Irlande, puis dans son village natal; Martin, derrière les barricades de sa villa dans la Creuse.

S'inscrivant dans la lignée de Houellebecq, à qui il a consacré un essai, le romancier Aurélien Bellanger a consacré son premier roman à la destinée d'un génie du numérique, Pascal Ertanger. De son enfance à sa mort, Ertanger ne cesse de ressentir une forme d'inadaptation au monde. Très jeune, il en préfère le reflet inversé dans la vitre de son Velux à son original, source de dangers et d'incompréhensions. Peu sociable au collège, il intègre la société informatique versaillaise, constituée d'«adolescents pionniers " de la révolution informatique, vivant encore « dans les marges de la société industrielle ", mais déjà " parfaitement adaptés pour survivre à l'ère du numérique ${ }^{34}$ ». Cet imaginaire du génie isolé, capable de révolutionner le monde sans sortir de sa chambre, séduit le jeune Ertanger au point qu'il finit lui-même par y coller : tout au long de sa fulgurante carrière, c'est toujours dans le retrait (dans sa chambre, la tour d'un manoir, une cabine de bateau, ou son propre corps transformé par ses acouphènes en boîte de résonance) qu'il puise l'inspiration et la force pour se lancer dans un nouveau projet qui, toujours, étendra le réseau: création d'un jeu, lancement de chânes de Minitel rose, démocratisation de l'accès à Internet, etc.

30 À l'opposé de ces quelques personnages qui fuient temporairement le monde pour se/le réinventer, d'autres s'abritent au contraire dans la pure consommation de fictions - « faiseurs » qui exploitent le réseau sans chercher à l'étendre. Ainsi en est-il du personnage de Mikki dans Mikki et le village miniature, de Mika Biermann ${ }^{35}$. Comme des Esseintes et Oblomov, Mikki est à l'abri du besoin, depuis l'héritage de ses parents. Un jour, entre deux épisodes de La Petite Maison dans la prairie dont il s'abrutit quotidiennement, il découvre dans la cave de la maison familiale un village miniature peuplé de personnages doués de vie, avec lesquels il tente vainement d'entrer en contact. Incapable, par manque de ressources imaginatives, d'assumer le rôle du dieu créateur qui lui est providentiellement dévolu, Mikki finit par détruire le village par dépit.

31 Un autre personnage de Féerie générale évoque le même type de trajectoire. Tsutomu Miyazaki est un otaku. Figure relativement tolérée dans la société nipponne, « le jeune otaku refuse l'affairisme ambiant ; il traîne en pantoufles l'air blasé, sort le moins possible, vit reclus parmi ses mangas et entretient dès les années 80 un rapport privilégié avec son écran de télé et son ordinateur ${ }^{36} »$. Garçon sans histoires, Miyazaki était un être passif, le pur réceptacle de fictions, jusqu'au jour où il assassine sauvagement quatre fillettes. Son retour au monde s'opère avec une extrême violence, comme si, à l'instar de Mikki, il n'avait pas eu les moyens de faire de sa retraite un projet créateur. 
De manière générale, si l'on reprend la comparaison avec nos figures du XIx ${ }^{e}$ siècle, on observe que les capacités imaginatives des individus sont bien plus valorisées dans le schéma contemporain. L'esthétisme décadent de des Esseintes, le goût d'Emma pour la fiction s'opposaient frontalement aux valeurs de leur temps. Au XxI siècle, la créativité est à ce point intégrée aux attentes de ce qui fait la grandeur des individus dans un monde en réseau, qu'elle opère comme une valeur de compromis $^{37}$. Fait marquant, à l'exception de Mikki, aucun des personnages que nous venons de croiser ne se situe sur le pôle critique. Ce qui les oppose, en termes de grandeur, c'est la qualité du compromis qu'ils frayent entre capacité à tisser des liens et mobilisation de ressources imaginatives. Ce qui rend tolérables les défections de Batoule et de Pascal Ertanger, c'est leur caractère temporaire, assorti de la promesse d'un retour créatif dans le monde. Ce qui rend misérable la figure de Mikki et criminelle celle de l'otaku, c'est le renversement en pure destruction du potentiel créateur dont on pouvait leur faire crédit. Sortir du monde en réseau requiert d'importantes ressources d'authenticité ainsi qu'un maillage suffisant. À nouveau, nous retrouvons ce paradoxe : s'arracher du système demande d'accumuler, ou d'avoir accumulé, ce qui en fait la grandeur.

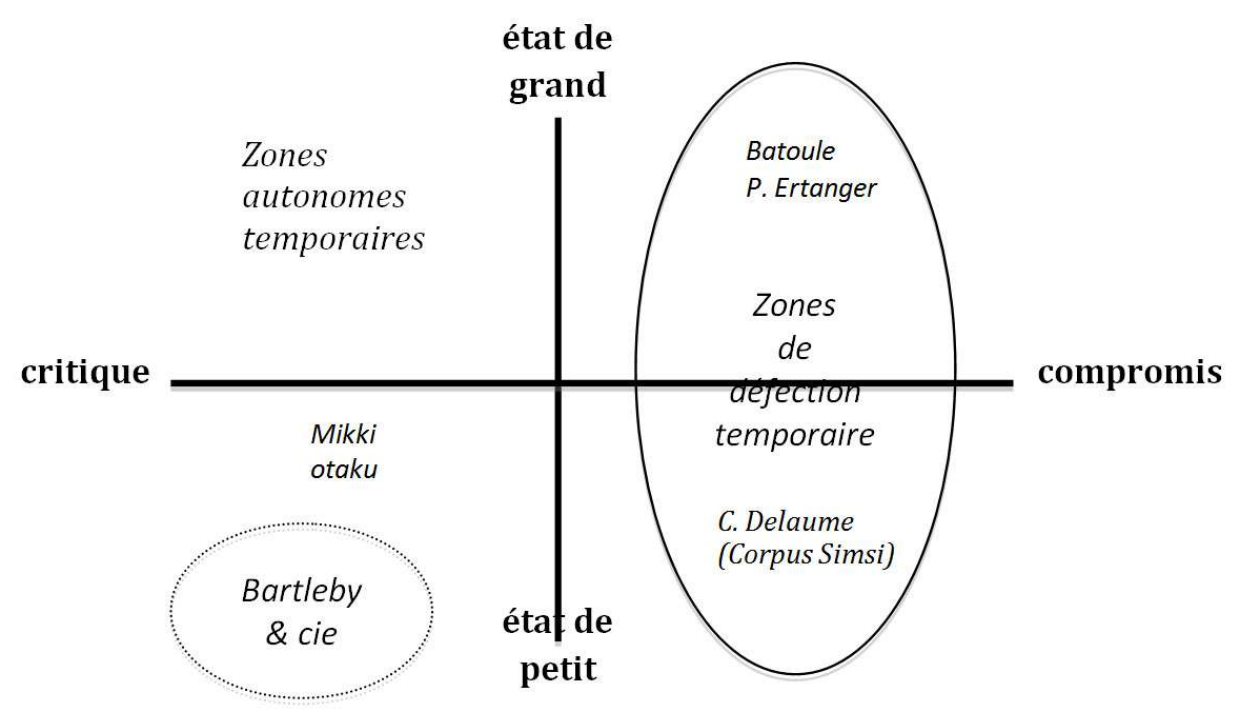

\subsection{Axe 1 : critique vs. compromis}

Si les sorties, provisoires et ambiguës, de ces personnages s'apparentent davantage à des formes de compromis, à quoi pourrait bien, dès lors, ressembler un authentique exit hors d'un monde en réseau? Nous sommes bien en peine de trouver des figures à la fois critiques et capables de se ménager une sortie par d'autres moyens que la négativité d'un Mikki. Le récent succès de la biographie de Christopher McCandless - grâce au récit de Jon Krakauer $^{38}$, mais surtout à l'adaptation cinématographique qu'en a faite Sean Penn fait même douter du caractère possible et viable d'un exit qui soit davantage qu'une zone de défection temporaire ou un fantasme de retour à un mode de vie pré-connexionniste.

Pour repeupler le pôle critique de notre schéma, il faut en réalité continuer à se tourner du côté du modèle des zones d'autonomie temporaire, qui continue de nourrir l'imagination littéraire. L'invention d'une position d'extériorité dans l'immanence du réseau est par exemple au cœur du roman de science-fiction anarcho-philosophique 
d'Alain Damasio, La Zone du dehors ${ }^{39}$. L'affaire Tarnac a été l'occasion pour des écrivains de se montrer sensibles au sort de Julien Coupat, mis en examen en 2008 suite à une spectaculaire opération anti-terroriste en Corrèze où ses amis et lui, soupçonnés de constituer la "cellule invisible » d'une mouvance " anarcho-autonome », habitaient une ferme ${ }^{40}$. Dans Tomates, Nathalie Quintane s'interroge cependant sur les limites d'un mouvement dont les appels et textes manifestaires redoublent leur splendide isolement d'un mimétisme du grand style insurrectionnel, qui vaut certes comme «forme de vie moins minable", mais fait un "pari sur l'avenir dans une langue qui est un pari sur le patrimoine $e^{41} »$.

La littérature contemporaine est en revanche extrêmement réceptive au personnage de Bartleby. Objet de nombreuses réappropriations, il fascine les philosophes, de Gilles Deleuze à Giorgio Agamben ${ }^{42}$, mais aussi les poètes et essayistes. Sous l'enseigne «Bartleby \& cie », Enrique Vila-Matas et Jean-Yves Jouannais rassemblent des artistes dont la posture consiste à ne plus produire ou à ne plus vendre. Ainsi Rimbaud, Beckett, Duchamp et Robert Walser côtoient-ils d'autres mutiques comme Félix Fénéon ou Jacques Vaché, dont l'influence littéraire est inversement proportionnelle à la quantité de leur production. La récente édition des lettres de refus d'Henri Michaux ${ }^{43}$, ainsi que l'essai que Maurizio Lazzarato consacre à Marchel Duchamp ${ }^{44}$, participent de cet intérêt pour les artistes qui aimeraient mieux pas.

Ce surinvestissement de la figure de Bartleby pose question. S'il témoigne d'une fascination de la littérature pour une certaine forme de grandeur dans la pénurie -« une pénurie qui serait le contraire d'une privation ${ }^{45} »$, il est aussi le signe d'une difficulté à penser la possibilité d'un exit au départ, non d'une double négation (le renversement d'une misère en grandeur), mais d'une authentique positivité critique. En d'autres termes, l'engouement pour Bartleby et, à travers lui, pour les formes de résistances à la littérature, n'est-il pas significatif d'un épuisement des formes de résistances en littérature?

\section{BIBLIOGRAPHY}

\section{Fuvres citées}

AMÉRIO (Sandy) (dir.), Storytelling. Index sensible pour agora non représentative, Paris, Aubervilliers, coéd. École nationale supérieure des beaux-arts, Fondation d'entreprise Ricard et les Laboratoires d'Aubervilliers, 2004.

ARLIX (Éric), Le Monde jou, Paris, Verticales, 2005.

BEIGBEDER (Frédéric), 5,90€, Paris, Le Livre de Poche, 2015 [99 francs, Grasset, 2000].

BELlANGER (Aurélien), La théorie de l'information, Paris, Folio, 2014 [Gallimard, 2012].

Biermann (Mika), Mikki et le Village miniature, Paris, P.O.L., 2015.

DAmasio (Alain), La Zone du Dehors, Paris, Cylibris, 1999.

TRANS-, $20 \mid 0000$ 
Delaume (Chloé), Corpus simsi, Paris, Léo Scheer, 2003.

FlaUbert (Gustave), Madame Bovary, Paris, Folio, 2004 [1857].

GLEIZE (Jean-Marie), Tarnac, un acte préparatoire, Paris, Éd. du Seuil, 2011.

GonTcharov (Ivan), Oblomov, trad. A. Adamov, Paris, Folio, 2007 [1859].

HouellebecQ (Michel), La Carte et le Territoire, Paris, Flammarion, 2010.

HUYSMANS (Joris-Karl), À rebours, Paris, Folio, 1983 [1884].

KRAKAUER (Jon), Into the wild. Voyage au bout de la solitude, trad. Ch. Molinier,

Paris, Presses de la Cité, 2008 [1996].

Melville (Herman), Bartleby le scribe, trad. P. Leyris, Paris, Folio, 1996 [1853].

Michaux (Henri), Donc, c'est non, éd. de J.-L. Outers, Paris, Gallimard, 2016.

NADAUD (Alain), D'écrire j'arrête, Paris, Tarabuste, 2010.

PEREC (Georges), Un homme qui dort, Paris, Denoël, 1967.

PireYre (Emmanuelle), Féérie générale, Paris, Points, 2013 [Éd. de l'Olivier, 2012].

Quintane (Nathalie), Tomates, Paris, Points, 2014 [P.O.L., 2010].

SAlVAYRe (Lydie), Portrait de l'écrivain en animal domestique, Paris, Éd. du Seuil, 2007.

Vila-MatAs (Enrique), Bartleby et compagnie, trad. É. Beaumatin, Paris, C. Bourgois, 2009.

\section{Ressources}

AgAmben (Giorgio), Bartleby ou la création, trad. de C. Walter, Paris, Circé, 2014.

BARRÈRE (Anne) et Martuccelli (Danilo), Le Roman comme laboratoire : De la connaissance

littéraire à l'imagination sociologique, Villeneuve d'Ascq, Presses Universitaires du Septentrion, 2009.

BerKMAn (Gisèle), L'effet Bartleby. Philosophes lecteurs, Paris, Hermann, 2011.

BERTRAND (Jean-Pierre), Biron (Michel), Dubois (Jacques), Paque (Jeannine), Le Roman célibataire, d’À rebours à Paludes, Paris, J. Corti, 1996.

BESSON (Anne), Constellations. Des mondes fictionnels à l'imaginaire contemporain, Paris, CNRS

Éditions, 2015.

BEY (Hakim), TAZ, zone autonome temporaire, trad. Ch. Tréguier, Paris, Éd. de l'Éclat, 1997 [1991].

BOLTANSKI (Luc) et Thévenot (Laurent), De la justification, Paris, Gallimard, 1991.

BOLTANSKI (Luc) et Chiapello (Ève), Le Nouvel Esprit du capitalisme, Paris, Gallimard, 1999.

LE BRETON (David), Disparaître de soi. Une tentation contemporaine, Paris, Métailié, 2015.

CITTON (Yves), Mythocratie : Storytelling et imaginaire de gauche, Paris, Éd. Amsterdam, 2010.

EHRENBERG (Alain), La fatigue d'être soi. Dépression et société, Paris, Odile Jacob, 1998.

FORTIN-TILLARD (Patrick), « L'effet-Bartleby : répétitions et nouveautés dans Un homme qui dort

TRANS-, $20 \mid 0000$ 
de Georges Perec ", Études littéraires, vol. 42, n² 2, 2011, p. 181-193, disponible sur :

http://id.erudit.org/iderudit/1011528ar.

HIRSCHMAN (Albert O.), Exit, voice, loyalty : Défection et prise de parole, trad. Cl. Besseyrias,

Bruxelles, Éditions de l'Université de Bruxelles, 2011 [1970].

JOUANNAIS (Jean-Yves), Artistes sans œuvres. I would prefer not to, Paris, Verticales, 2009 [Hazan,

1997].

LAVocat (Françoise), Fait et fiction, Paris, Éd. du Seuil, 2016.

LAZZARATo (Maurizio), Marcel Duchamp et le refus du travail, Paris, Les Prairies Ordinaires, 2014.

MASSERA (Jean-Charles), «It's too late to say littérature (Aujourd'hui recherche formes

désespérément) », Revue Ah!, n 10, 2010, p. 7-45.

Revue Multitudes, Majeure «Contre-fictions politiques », $\mathrm{n}^{\circ}$ 48, 2012, p. 70-146.

SALMON (Christian), Storytelling : La Machine à fabriquer des histoires et à formater les esprits,

Paris, La Découverte, 2007.

SMITH (Frank), Fonctions Bartleby, bref traité d’investigations poétiques, Paris, Le Feu sacré, 2015.

VIART (Dominique), « Historicité de la littérature contemporaine », in VIART (D.) et DEMANZE (L.)

(dir.), Fins de la littérature. Historicité de la littérature contemporaine, t.II, Paris, Armand Colin,

2012.

\section{NOTES}

1. Jean-Charles Massera, «It's Too Late to Say Littérature (Aujourd'hui recherche formes désespérément) », revue Ah!, $\mathrm{n}^{\circ} 10,2010$, p. 38.

2. Christian Salmon, Storytelling : La Machine à fabriquer des histoires et à formater les esprits, Paris, La Découverte, 2007.

3. Luc Boltanski et Ève Chiapello, Le Nouvel Esprit du capitalisme, Paris, Gallimard, 1999.

4. Luc Boltanski et Ève Chiapello, op. cit., p. 81-86.

5. Yves Citton, Mythocratie: Storytelling et imaginaire de gauche, Paris, Amsterdam, 2010.

6. Christian Salmon, op. cit., p. 103.

7. Anne Besson, Constellations. Des mondes fictionnels à l'imaginaire contemporain, Paris, CNRS Éditions, 2015.

8. Albert O. Hirschman, Exit, voice, loyalty: Défection et prise de parole, trad. fr. Claude Besseyrias, Bruxelles, Éditions de l'Université de Bruxelles, 2011 [1970].

9. Sandy Amério, Storytelling. Index sensible pour agora non représentative, coédition École nationale supérieure des beaux-arts, Fondation d'entreprise Ricard et Laboratoires d'Aubervilliers, 2004 ; Éric Arlix, Le Monde jou, Paris, Verticales, 2005 ; Lydie Salvayre, Portrait de l'écrivain en animal domestique, Paris, Seuil, 2007.

10. Un exemple : Alain Nadaud, D'écrire j'arrête, Paris, Tarabuste, 2010.

11. Dominique Viart observe, depuis les années 1980, un regain d'intérêt pour la forme du récit dans la littérature française. Voir Dominique Viart, «Historicité de la littérature contemporaine ", in Dominique Viart et Laurent Demanze (dir.), Fins de la littérature. Historicité de la littérature contemporaine, tome II, Paris, Armand Colin, 2012. 
12. Revue Multitudes, Majeure « Contre-fictions politiques », 2012, ${ }^{\circ} 48$, p. 70-146.

13. Chloé Delaume, Corpus simsi, Paris, Léo Scheer, 2003, p. 106.

14. Chloé Delaume, op. cit., p. 42.

15. Delaume semble adhérer à ce que Françoise Lavocat qualifie de «définition moniste » de la fiction. Pour Lavocat, cette conception repose sur une acception négative et dépassée de la fiction, en en faisant une forme de leurre quotidien et efficace. On retrouverait ce monisme chez plusieurs auteurs (Christian Salmon, Yves Citton, Nancy Huston) qui craignent ou diagnostiquent une fictionnalisation $\mathrm{du}$ monde et des rapports humains, notamment sous l'influence $\mathrm{du}$ storytelling (voir Françoise Lavocat, Fait et fiction, Paris, Seuil, 2016, p. 41-56).

16. Frédéric Beigbeder, 5,90€, Paris, Le Livre de Poche, 2015 [99 francs, Grasset, 2000], p. 20-21.

17. Ces figures sont tirées d'un corpus en cours d'élaboration dans le cadre d'un projet de recherche (PDR) intitulé «Storyfic». Financé par le F.R.S.-FNRS belge, le projet vise à évaluer l'impact du phénomène du storytelling sur l'écriture de fiction contemporaine.

18. Jean-Yves Jouannais, Artistes sans œuvres. I would prefer not to, Paris, Verticales, 2009 [Hazan, 1997]; Enrique Vila-Matas, Bartleby et compagnie, trad. fr. Éric Beaumatin, Paris, Christian Bourgois, 2009; Giorgio Agamben, Bartleby ou la création, trad. fr. Carole Walter, Paris, Circé, 2014 ; Frank Smith, Fonctions Bartleby, bref traité d'investigations poétiques, Paris, Le Feu sacré, 2015.

19. Le terme geek a été adopté, en français, depuis les années 2000 pour désigner un ensemble de consommateurs culturels, rassemblés par des pratiques et passions communes: goût pour les jeux vidéo, fascination pour les nouvelles technologies ou encore intérêt particulier pour les genres de la fantasy et de la science-fiction. L'appellation de geek fédère ces individus sous une identité commune, aujourd'hui valorisée.

Il n'en a pas toujours été ainsi. Le terme nerd, désignait, depuis les années 50, le stéréotype de l'informaticien fermé sur lui-même, handicapé par son hyper-spécialisation qui l'empêcherait de nouer des relations, si ce n'est avec des machines ou par leur intermédiaire. C'est le même genre d'individu que stigmatisait le terme nolife: des personnes qui se consacrent tellement à leur travail ou à leur passion qu'elles en oublieraient que « la vie » est ailleurs que derrière un écran. Les termes nerd et nolife peuvent donc être considérés comme d'anciennes dénominations pour un rôle social que l'imaginaire capitaliste contemporain aurait réintégré et revalorisé sous les traits du geek (voir Anne Besson, «Glossaire » in Constellations. Des mondes fictionnels à l'imaginaire contemporain, Paris, CNRS Éditions, 2015).

20. Hakim Bey, TAZ, zone autonome temporaire, trad. fr. Christine Tréguier, Paris, Éditions de l'Éclat, 1997 [1991]. Hakim Bey est le pseudonyme de l'essayiste américain Peter Lamborn Wilson.

21. Luc Boltanski et Laurent Thévenot, De la justification, Paris, Gallimard, 1991, p. 338-339.

22. Ivan Gontcharov, Oblomov, trad. fr. Arthur Adamov, Paris, Folio, 2007 [1859], p. 135-142.

23. Luc Boltanski et Laurent Thévenot, op. cit.

24. Luc Boltanski et Ève Chiapello, op. cit., p. 54-55.

25. Joris-Karl Huysmans, À rebours, Paris, Folio, 1983 [1884], p. 150-151.

26. Jean-Pierre Bertrand, Michel Biron, Jacques Dubois, Jeannine Paque, Le Roman célibataire, d’À rebours à Paludes, Paris, José Corti, 1996.

27. Georges Perec, «Le bonheur est un processus... on ne peut pas s'arrêter d'être heureux ", propos recueillis par Marcel Benabou et Bruno Marcenac (1965), cités par Patrick Fortin-Tillard, « L'effet-Bartleby : répétitions et nouveautés dans Un homme qui dort de Georges Perec ", Études littéraires, vol. 42, $\mathrm{n}^{\circ}$ 2, 2011, p. 185. Notons que le texte de Perec atteste de cet intérêt pour la nouvelle de Melville, puisqu'il y renvoie explicitement; voir Georges Perec, Un homme qui dort, Paris, Denoël, 1967, p. 152-153.

28. Luc Boltanski et Ève Chiapello, op. cit., p. 168.

29. Alain Ehrenberg, La Fatigue d'être soi : Dépression et société, Paris, Odile Jacob, 1998, p. 15. 
30. David Le Breton, Disparaître de soi. Une tentation contemporaine, Paris, Métailié, 2015. Au niveau de l'entreprise, la sociologie clinique (Christophe Dejours, Vincent de Gaulejac) dénonce, depuis plus de vingt ans, l'emprise des nouvelles techniques de management sur les individus.

31. Anne Barrère et Danilo Martuccelli, Le Roman comme laboratoire : De la connaisance littéraire à l'imagination sociologique, Villeneuve d'Ascq, Presses Universitaires du Septentrion, 2009.

32. Luc Boltanski et Ève Chiapello, op. cit., p. 438-439.

33. Emmanuelle Pireyre, Féérie générale, Paris, Points, 2013 [Éditions de l'Olivier, 2012], p. 158.

34. Aurélien Bellanger, La Théorie de l'information, Paris, Folio, 2014 [Gallimard, 2012], p. 78.

35. Mika Biermann, Mikki et le Village miniature, Paris, P.O.L., 2015.

36. Emmanuelle Pireyre, op. cit., p. 22.

37. Luc Boltanski et Ève Chiapello, op. cit., p. 192.

38. Jon Krakauer, Into the wild. Voyage au bout de la solitude, trad. fr. Christian Molinier, Paris, Presses de la Cité, 2008 [1996].

39. Alain Damasio, La Zone du Dehors, Paris, Cylibris, 1999.

40. Jean-Marie Gleize, Tarnac, un acte préparatoire, Paris, Seuil, 2011.

41. Nathalie Quintane, Tomates, Paris, Points, 2014 [P.O.L., 2010], p. 47 et 108.

42. Gisèle Berkman, L'effet Bartleby : Philosophes lecteurs, Paris, Hermann, 2011.

43. Henri Michaux, Donc, c'est non, éd. Jean-Luc Outers, Paris, Gallimard, 2016.

44. Maurizio Lazzarato, Marcel Duchamp et le refus du travail, Paris, Les Prairies Ordinaires, 2014.

45. Jean-Yves Jouannais, Artistes sans cuvres, Paris, Verticales, 2009, p. 115.

\section{ABSTRACTS}

Concerned by the exploitation of narrative and fiction for commercial or strategic purposes, many writers and observers wonder what power literature still has today, when the logic of capitalism took over its tools and seems always one step ahead of its critical potentialities. To use Albert Hirschman's terminology, if the possibilities of voice seem exhausted, can the same be said of the exit strategy, in a networked world precisely fortified by the constant injunction to freedom and autonomy? Moving the issue to the field of the novel, our contribution focuses on some contemporary characters' wish to withdraw (geeks, secluded artists, autonomous activists, hermits of the virtual age, etc.), compared to emblematic literary figures of exit in the XIXth century (des Esseintes, Bartleby, Oblomov, Emma Bovary), so as to measure the changes that affected the very space of possibilities of exit, and their consequences for forms of resistance in literature.

Inquiets de l'instrumentalisation du récit et de la fiction à des fins mercantiles ou stratégiques, de nombreux écrivains et observateurs s'interrogent aujourd'hui : quels pouvoirs reste-t-il à la littérature, quand la logique capitaliste s'est appropriée ses outils et semble toujours en avance sur ses potentialités critiques? Pour reprendre la terminologie d'Albert Hirschman, si les modalités de contestation (voice) semblent épuisées, qu'en est-il des possibilités de défection (exit ), dans un monde en réseau qui se nourrit précisément de l'injonction permanente à la liberté et l'autonomie ? Déplaçant cette question sur le terrain romanesque, notre contribution s'intéresse à la volonté de retrait de quelques personnages contemporains (geeks, artistes reclus, militants autonomes, ermites des mondes virtuels, etc.), comparés à des figures littéraires emblématiques de la défection au XIX ${ }^{\mathrm{e}}$ siècle (des Esseintes, Bartleby, Oblomov, Emma Bovary), de manière à 
mesurer les changements qui ont affecté l'espace même de possibilités de fuite, et leurs conséquences pour des formes de résistance en littérature.

\section{AUTHORS}

\section{JUSTINE HUPPE}

Titulaire d'un Master en langues et littératures françaises et romanes, Justine Huppe est doctorante à l'université de Liège, dans le cadre du projet de recherche PDR (F.R.S.-FNRS) STORYFIC.

\section{FRÉDÉRIC CLAISSE}

Docteur en sciences politiques et sociales, Frédéric Claisse est chercheur post-doctorant à l'université de Liège. Ses enseignements et ses recherches portent sur l'épistémologie des sciences sociales et des méthodes participatives ; les interactions entre science, technologie et société ; la prospective et les visions du futur. Après une thèse sur la capacité du récit et de la fiction à configurer des dossiers complexes, il travaille sur les effets du storytelling sur le roman contemporain dans le cadre du projet de recherche PDR (F.R.S.-FNRS) STORYFIC. 